Results In 2005 there were 1046 new patients and 3752 follow ups In 2009 there were 1227 new patients and 3275 follow ups, 535 of which were seen by the telephone clinic services exclusively. There were no adverse events recorded in this cohort.

The number of follow ups reduced substantially saving clinic time, improving efficiency and allowing us to see extra $17.3 \%$ patients.

Conclusion The nurse led telephone clinics have improved efficiency, safety of the gastroenterology services and improved the new to follow up ratio from 0.27 to 0.37 . Patient survey data suggests that they were not only happy with the telephone clinic service but would prefer that in order to save time and money.

Disclosure of Interest None Declared.

\section{PTH-164 WHAT ISSUES NEED TO BE ADDRESSED REGARDING WORKFORCE PLANNING IN RELATION TO THE CHANGING DEMOGRAPHICS OF DOCTORS WITHIN THE FIELD OF GASTROENTEROLOGY?}

doi:10.1136/gutjnl-2013-304907.651

1."R Preedy, 'M McStay. 'Gastroenterology, Colchester General Hospital, Colchester, UK

Introduction Many challenges lay ahead in the future development of the NHS including the changing workforce demographic, tighter funding and the prospect of increasing demands on services. Currently in the specialty of Gastroenterology $15 \%$ of consultants and $34 \%$ of trainees are female. To help realise the problems faced in workforce planning by this change of demographic we have tried to identify the current working patterns and perceptions of satisfaction and work-life balance amongst female Consultant Gastroenterologists.

Methods A survey was developed using previously validated questions, these were then piloted amongst a group of female hospital doctors to ensure that they were fit for purpose. A total of 110 Female Gastroenterologists were then emailed with a web based link to the survey along with information explaining what the survey was for. The responses were managed by RAND Europe, an independent non-profit organisation who have experience of similar projects.

Results There was a $63 \%$ response rate. The majority of respondents were between the ages of 41 to 50, and highly experienced of the NHS having worked for at least 15 years. $90 \%$ had achieved some form of higher degree, but only $2 \%$ worked in academic posts. $76 \%$ of respondents had had children, $73 \%$ of those felt that they provided the majority of childcare in comparison to their partner. $48 \%$ had delayed having children until they had become a consultant and $41 \%$ felt that they had had fewer children because of their career. A minority felt that their gender and parental status had negatively affected their career. The majority of respondents worked long hours with $30 \%$ working more than 49 hours per week. $11 \%$ of respondents worked part time, 19\% were aware of a flexible working policy but not able to use it while $35 \%$ were not aware that one existed. Only 38\% percent were satisfied with the recognition they got for their work. Almost $20 \%$ had experienced bullying from either mangers or other colleagues in the preceding 12 months and $27 \%$ had received harassment from patients or patient's relatives. Almost 45\% felt more stressed than usual and that their work life had a negative impact on their life. Although the majority of respondents felt happy with their current lifestyle, 10\% were dissatisfied with their life, and $27 \%$ would change it if they could.

Conclusion Current female Gastroenertologists are competently educated but underrepresented in academic posts. They are more likely to be the main care giver at home but face inflexible working arrangements. They reported high levels of bullying as well as a perceived lack of value from their NHS employer. To maintain a committed and motivated medical workforce the issues highlighted in this survey will need to be addressed in future workforce planning. Disclosure of Interest None Declared.

\section{PTH-165 VALIDATING THE COLONOSCOPY WAITING LIST - CHALLENGING BUT REWARDING}

doi:10.1136/gutjnl-2013-304907.652

1."R Prudham, ' ${ }^{1}$ B Boyer. ${ }^{1}$ Gastroenterology, Pennine Acute NHS Trust, Bury, UK

Introduction Endoscopy services within the UK are under pressure with increasing demand predicted and a need to maintain timeliness. The Department of Health for England and Wales wishes to improve outcomes for colorectal cancer and so increase the volume of lower GI endoscopy. Endoscopy services must increase efficiency to meet this challenge. We felt there was an opportunity to increase quality and reduce variation by ensuring all scheduled colonoscopies complied with National and departmental guidance. This is a key quality standard of the Global Rating Scale. We identified sessions in job plans of 3 nurse endoscopists within our trust. We then identified the guidance, informed endoscopists and the management team of the exercise we were undertaking and finally evaluated the intervention to be shared within our directorate team.

Methods Patients awaiting scheduled colonoscopy were identified form the Primary Targetted List database held by the trust to manage waiting and scheduled patients. Patients being followed up for colorectal cancer were excluded from the process and study as there was no single protocol being followed at the time of the study. The patient groups studied were therefore those on surveillance pathways for polyp follow up, family history or inflammatory bowel disease.

Clinical teams within the trust were informed by the clinical director of the process and that BSG guidelines for the conditions listed above were to be followed. The initial phase of the process was undertaken by a single Nurse Endoscopist. Following validation against guidelines, the case was reviewed by the clinical director and a letter addressed to the patient and copied to the patient's GP and secondary care consultant. The letter was co-signed by the nurse and the clinical director.

Following the establishment of the process as feasible, two other nurse endoscopists were trained in validating the procedures. Sessions were identified in job plans to ensure this process could be perpetuated long term.

\section{Results}

\section{Abstract PTH-165 Table 1}

\begin{tabular}{lccccc}
\hline 2012 & Total & Canc & Defer & Leave & Other \\
\hline Apr & 102 & 42 & 18 & 39 & 3 \\
May & 96 & 28 & 7 & 60 & 1 \\
Jun & 106 & 28 & 15 & 63 & 0 \\
July & 124 & 43 & 22 & 59 & 0 \\
Aug & 76 & 27 & 10 & 37 & 2 \\
Sept & 95 & 34 & 8 & 52 & 1 \\
Oct & 102 & 36 & 13 & 53 & 0 \\
Total & 701 & 238 & 93 & 363 & 6 \\
\hline
\end{tabular}

Large numbers of surveillance colonoscopies are undertaken (73124 per month). Many were listed on basis of colonoscopy findings and not checked for histology. A wide range of clinicians including non-endoscopists were listing patients. Guidelines were not adhered too. There was resistance to this standardisation from clinicians, GPs and patients initially.

Conclusion Wide variations in practise were observed. A large number of unnecessary colonoscopies were deferred to a more appropriate interval, producing benefits in quality and efficiency using existing resources.

Disclosure of Interest None Declared.

\section{REFERENCE}

BSG (2010) Guidelines for colorectal cancer screening and surveillance in moderate and high risk groups BSG (2002) Surveillance following Adenoma Removal 